\title{
Compressed Sensing Inspired Line Feature Detection for In-Situ Transmission Electron Microscopy
}

\author{
Haoyang Ni1, Aram Yoon2 and Jian-Min Zuo3
}

1University of Illinois at Urbana-Champaign, United States, 2Center for Multidimensional Carbon Materials, Institute for Basic Science (IBS), Ulsan 44919, Republic of Korea, United States, 3University of Illinois at Urbana-Champaign, Urbana, Illinois, United States

Line features captured in transmission electron microscope (TEM) images are often from the diffraction contrast of dislocations, grain boundaries and interfaces. The motion of these lines is the direct indicator of the materials response to the driving forces, and thus specific information about the physical and chemical processes happening in materials can be obtained by analyzing the line features, such as location, direction and curvature, and their change with respect to time. Automatic identification and quantification of line features, therefore, are increasingly important to fully comprehend the dataset acquired by the in-situ TEM, whose size is often too large to analyze in a manual way.

Here, we present a semi-automated framework of line detection and feature extraction inspired by the compressed sensing based image reconstruction approach, which was used to remove the stripe-like artifacts in electron microscopy images [1]. In Fourier space, line features present as wedge-shape signals. We masked out the signal with the wedge filter and the real space image can be reconstructed with the missing wedge by total-variation minimization. By taking the difference between the reconstructed and as-recorded images, line features are enhanced and then detected after applying intensity and length threshold. Finally, skeletonizing the line features can produce the detected lines down to pixel-scale precision.

To illustrate the implementation and performance of our image processing framework, we used the example of crystallographic shear planes (CSPs) defects in a TiO2 nanocrystal captured in a video of the dark-field TEM images. The nanocrystal was exposed to the controlled $\mathrm{O} 2$ environment and was being oxidized in our presented dataset. The video was first preprocessed using binning, drift-correction and frame-wise averaging for an effective 1 frame-per-second (FPS) rate. A Gaussian filter was also applied to smooth the images. Figure 1 compares our result with the application of Sobel filter and Canny edge detector, which are the common methods for line detection. Our result shows better accuracy compared to these two methods.

To illustrate the robustness of our method, we analyzed the entire video set in an automated way. Figure 2 shows the results for frames 1, 20 and 40, respectively, from the dataset, where the algorithm correctly identifies the split of CSP lines ( $a$ and b), which merge in the last frame (c). Once the lines are detected, the slope of each point on the CSP line, as well as the line length, can be calculated. By utilizing every frame in the dataset, it is possible to map the CSP defect transition quantitively to provide the full picture of the dynamic process.

While our algorithm proves to be efficient, the accuracy of line detection depends highly on the image contrast around the lines and edges. In the case of dark-field TEM imaging, the mixed contrast often shadows and smears the lines and edges. However, by following the time series, the algorithm is still able to provide scientific insights in a statistical way due to having enough sample points in both the time and space domains. To summarize, we presented an algorithm for the line feature detection and quantification for in-situ datasets with the enhanced accuracy. Further generalization of our algorithm in different application scenarios, such as dislocation line defect detection, will enable their quantitative analysis, where density and curvature are both important [2]. 


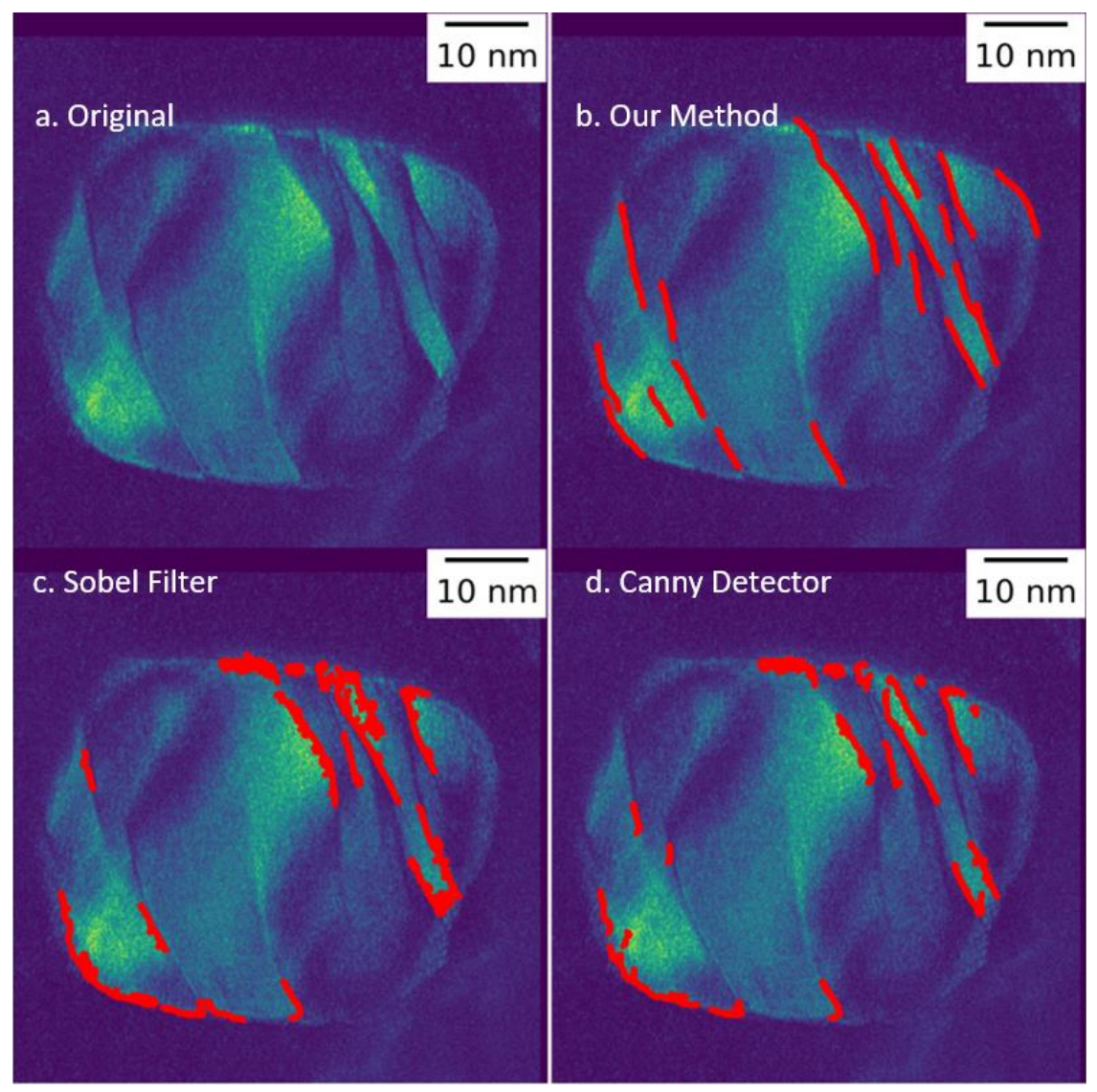

Figure 1. Figure. 1 Comparison of line detection methods. a. Original Image. b. CSP lines detected by our method c. CSP lines detected by Sobel filter and intensity threshold d. CSP lines detected by Canny edge detector

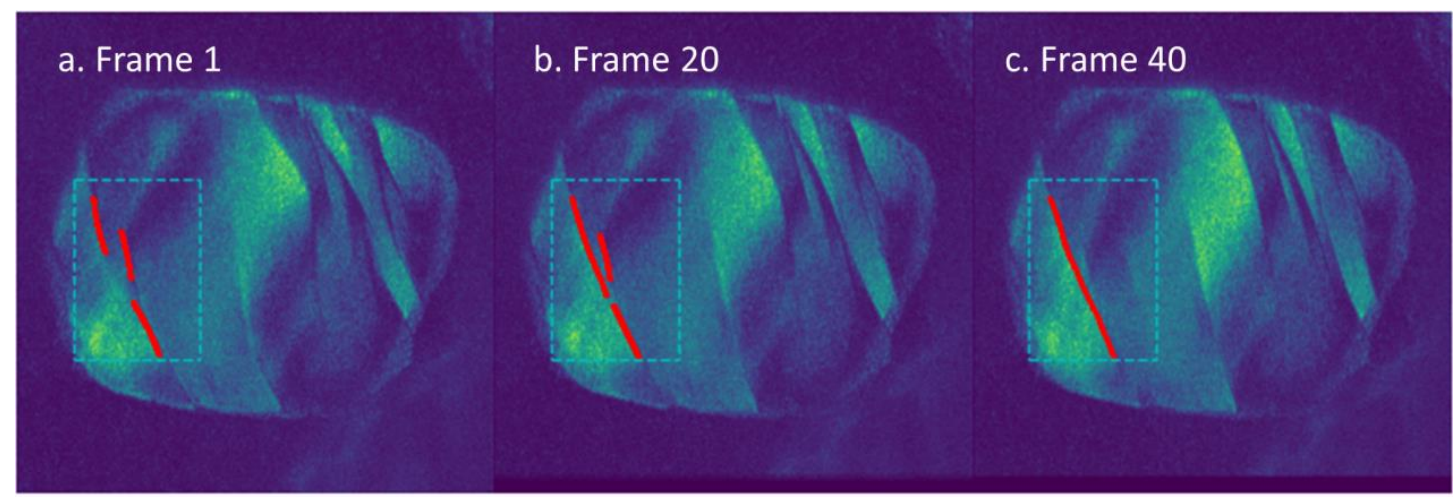

Figure 2. Figure 2. CSP detection within ROI (cyan). CSP line segments merges as oxidation reaction continues.

\section{References}

[1] Schwartz, J., Jiang, Y., Wang, Y., Aiello, A., Bhattacharya, P., Yuan, H., Mi, Z., Bassim, N. \& Hovden, R. (2019). Removing Stripes, Scratches, and Curtaining with Non-Recoverable Compressed Sensing. Microscopy and Microanalysis 25, 705-710.

[2] Work supported by Strategic Research Initiative of College of Engineering, University of Illinois UrbanaChampaign 